\title{
Literature Review of Machine-Learning Algorithms for Pressure Ulcer Prevention: Challenges and Opportunities
}

\author{
Fernando Ribeiro*(D), Filipe Fidalgo, Arlindo Silva $\mathbb{D}^{D}$, José Metrôlho $\mathbb{D}^{D}$, Osvaldo Santos and Rogério Dionisio \\ R\&D Unit in Digital Services, Applications and Content, Polytechnic Institute of Castelo Branco, \\ 6000-767 Castelo Branco, Portugal; ffidalgo@ipcb.pt (F.F.); arlindo@ipcb.pt (A.S.); metrolho@ipcb.pt (J.M.); \\ oas@ipcb.pt (O.S.); rdionisio@ipcb.pt (R.D.) \\ * Correspondence: fribeiro@ipcb.pt; Tel.: +351-272-339-300
}

Citation: Ribeiro, F.; Fidalgo, F.; Silva, A.; Metrôlho, J.; Santos, O.; Dionisio, R. Literature Review of Machine-Learning Algorithms for Pressure Ulcer Prevention: Challenges and Opportunities. Informatics 2021, 8, 76. https:// doi.org/10.3390/informatics8040076

Academic Editor: You Chen

Received: 15 October 2021

Accepted: 8 November 2021

Published: 10 November 2021

Publisher's Note: MDPI stays neutral with regard to jurisdictional claims in published maps and institutional affiliations.

Copyright: (c) 2021 by the authors. Licensee MDPI, Basel, Switzerland. This article is an open access article distributed under the terms and conditions of the Creative Commons Attribution (CC BY) license (https:/ / creativecommons.org/licenses/by/ $4.0 /)$.

\begin{abstract}
Pressure ulcers are associated with significant morbidity, resulting in a decreased quality of life for the patient, and contributing to healthcare professional burnout, as well as an increase of health service costs. Their prompt diagnosis and treatment are important, and several studies have proposed solutions to help healthcare professionals in this process. This work analyzes studies that use machine-learning algorithms for risk assessment and management of preventive treatments for pressure ulcers. More specifically, it focuses on the use of machine-learning algorithms that combine information from intrinsic and extrinsic pressure-ulcer predisposing factors to produce recommendations/alerts to healthcare professionals. The review includes articles published from January 2010 to June 2021. From 60 records screened, seven articles were analyzed in full-text form. The results show that most of the proposed algorithms do not use information related to both intrinsic and extrinsic predisposing factors and that many of the approaches separately address one of the following three components: data acquisition; data analysis, and production of complementary support to well-informed clinical decision-making. Additionally, only a few studies describe in detail the outputs of the algorithm, such as alerts and recommendations, without assessing their impacts on healthcare professionals' activities.
\end{abstract}

Keywords: artificial intelligence; burnout; clinical decision support; literature review; machine learning; pressure injury prevention; pressure ulcers prevention; quality of healthcare

\section{Introduction}

Quality of healthcare is the most important goal, not only for patients, but also for healthcare professionals. Particularly, in pressure ulcers (PU), predisposing factors defined as intrinsic (i.e., limited mobility, poor nutrition, comorbidities, aging skin) [1-4] and extrinsic (i.e., pressure from hard surfaces (e.g., bed, stretcher), shearing from involuntary muscle movements, friction from the patient's inability to move well in bed, excessive moisture) can influence healthcare quality [2,5]. Using these indicators objectively relies on the healthcare professional's perception of patient outcome (direct observation), which is based on how they rate the patients (i.e., using the Braden scale, the Waterlow scale, or the Norton risk-assessment scale for adults; and the Braden Q scale for children) [4]. Acquiring this data usually depends exclusively on human intervention. Recently, researchers have been exploring ways to integrate machine learning (ML) and the internet of things (IoT) into healthcare systems, to produce indicators/alerts useful to healthcare professionals [6-12]. Healthcare professionals cannot care for PU patients based only on predisposing factors, nor based only on humanless data. Posture recognition can identify when localized parts of the body are under continuous pressure, such as when patients are recovering from surgery, so blood supply to those areas is hindered and a pressure sore develops consequently [2,5-12]. For example, after identifying that a bedridden pressure ulcer patient is in the same position for a specific amount of time, based on sensors attached in the mattress (humanless data acquisition), the adequate time to change his position may differ based on gender, age, 
comorbidities, and other aspects (human intervention data acquisition). So, both types of data acquisition influence care quality, and are required to create evidence-based guidelines for preventing pressure ulcer development. This data combined with IoT and ML can provide systems to support caregivers' activities, avoiding unnecessary overburdening and/or caregiver burnout.

Worldwide, as far as the authors have knowledge, no studies were found that have considered these two types of data acquisition - healthcare professionals data acquisition and humanless data acquisition. Moreover, only a few studies managed effectively to produce potential recommendations/alerts to support professionals' activity.

This study aimed to analyze the association between ML and IoT in healthcare systems that produce indicators/alerts to healthcare professionals, simultaneously minimizing the burnout in healthcare professionals and improving pressure-ulcer patient care quality and safety.

This paper is organized as follows. Section 2 details the comparison with previous reviews. Section 3 presents the details of the methodology of the systematic literature review. Section 4 provides the data extraction and data analysis. Section 5 reports the discussion and findings. Section 6 presents a discussion about challenges and opportunities. Section 7 describes the strengths and limitations of this work. Finally, Section 8 presents the conclusions and directions for future work.

\section{Comparison with Previous Reviews}

In recent years, several studies have reviewed works related to the use of algorithms and models for PU prevention. From a comprehensive search, we found five previous systematic reviews related to this work. Tables 1 and 2 summarize some of their findings.

Table 1. Studies reviewing ML algorithms for PU prevention: year, focus, and timespan.

\begin{tabular}{|c|c|c|c|c|}
\hline Review & Year & Focus & Studies & Timespan \\
\hline [13] & 2021 & $\begin{array}{l}\text { Analyze the use of ML technologies in PU } \\
\text { management, identify their strengths } \\
\text { and weaknesses. }\end{array}$ & 32 & 2007-2020 \\
\hline [14] & 2019 & $\begin{array}{l}\text { Describe empirically derived models (risk assessment } \\
\text { tools) for predicting pressure-ulcer risk, assess the } \\
\text { predictive performance of these models, and evaluate } \\
\text { their clinical impact in reducing PU incidence. }\end{array}$ & 24 & 1996-2017 \\
\hline [15] & 2020 & $\begin{array}{l}\text { Understand and compare the applications of ML in } \\
\text { the care of diabetic foot-ulcer patients and compare } \\
\text { their diagnostic and prognostic accuracy. }\end{array}$ & 37 & 2010-2020 \\
\hline [16] & 2015 & $\begin{array}{l}\text { Identify the state of art of the approaches that use } \\
\text { software that provides relevant information based on } \\
\text { monitoring a patient's risk factor, to assist health } \\
\text { professionals in PU prevention support. }\end{array}$ & 36 & 1989-2014 \\
\hline [17] & 2020 & $\begin{array}{l}\text { Identify the outcomes from nurses when using } \\
\text { support systems on clinical decision-making for } \\
\text { PU management. }\end{array}$ & 16 & 1995-2017 \\
\hline
\end{tabular}


Table 2. Studies reviewing ML algorithms for PU prevention: results, opportunities, and future research.

\begin{tabular}{cc}
\hline Review & Analysis/Results \\
\hline & Studies were classified and organized into three groups: \\
& $12(38 \%)$ reported using ML technologies to develop \\
predictive models to identify risk factors, $11(34 \%)$ \\
reported using them in posture detection and \\
recognition, and $9(28 \%)$ reported using them in image \\
analysis for tissue classification and measurement of \\
PU wounds.
\end{tabular}

The characteristics of the included studies and models calibration) measurement statistics are combined for relevant models.
[14] are summarized, and performance (discrimination and

Identified Opportunities and Future Research

Apply these technologies on a large scale with clinical

data to further verify and improve their effectiveness, as well as to improve methodological quality. The prevention of PU was studied under different approaches; however, they are related and should be studied together. For example: ML technologies on predictive model and posture recognition need feedback from PU wound image analysis to improve their performance.

Validate the prognostic models for predicting ulcer risk and evaluate the clinical impact of empirically derived models.

For the development of more applicable ML algorithms, future research should compare the use of ML applications with current standards for care, health economic analyses, and large-scale data collection.

Whilst varying across several ML algorithms, all studies reported at least $90 \%$ accuracy. Applications where ML [15] had positive effects on diabetic foot-ulcer data analysis and outcomes include image segmentation and classification, raw data analysis, and risk assessment.
Most of the approaches use sensors to monitor the

patient's exposure to pressure, temperature, and humidity to generate reports regarding the intensity of each one of these risk factors, as well as the patient's [16] position in bed. Some approaches perform automated management of the risk factors using ventilation tubes and mattresses with porous cells to decrease the body's temperature and cause movable cells to automatically redistribute the pressure over the body.
Perform randomized control trials to verify which approaches are effective to reduce PU incidence and to verify which information provided by each of the approach is relevant to health professionals to support them on PU prevention.
All the analyzed studies describe knowledge-based systems that assessed the effects on clinical decision-making, clinical effects secondary to clinical decision support system use, or factors that influenced the use, or intention to use, clinical decision support systems by health professionals and the success of their implementation in nursing practice.

\begin{abstract}
Carry out studies that prioritize better adoption and interaction of nurses with clinical decision support systems, as well as studies with a representative sample of healthcare professionals.
\end{abstract}

To the best of our knowledge, there are no systematic literature review studies that evaluate how ML technologies are being used in PU prevention and provide recommendations and warnings to healthcare professionals. Some studies focused on the analysis of algorithms or models for predicting PU risk, usually having an associated risk indicator as an output (e.g., [14]). The study presented in [13] partially addresses this issue. In this study, the authors analyze works that used ML technologies with the aim of contributing to lessen the burden on medical staff by improving the prognosis and diagnostic accuracy of PU. Studies included an analysis of patients' electronic health records and developed a prediction model via data-mining techniques. Typically, the goals of these studies were to identify PU risk factors, so that health professionals could take customized preventive measures or compare different algorithm performances and interpretability in constructing a predictive model. The work presented in [15] analyzes the use of ML algorithms in diabetic foot ulcers. It focuses on a specific type of ulcer, by evaluating the accuracy of the ML algorithms. The work presented in [16] has some similarities with the work presented in this article, since it focuses on analyzing approaches that use software for monitoring the patient's risk factor and providing information to assist health professionals in PU prevention support. The analyzed works were published between 1989 and 2014, and most of them focus on proposing solutions for monitoring the patient's body position over time 
or monitoring parameters such as humidity, temperature, or blood flow. Additionally, they are not focused on approaches that use ML nor on the evaluation of its accuracy.

\section{Methodology}

This section presents a systematic review of studies that addressed the use of ML algorithms for risk assessment and management of preventive treatments of PU in bedridden patients. The purpose of this analysis is to identify the inputs considered in the algorithms and to get information on the strengths and weaknesses of each approach or algorithm and the achieved results.

The review is reported according to the Preferred Reporting Items for Systematic Reviews and Meta-Analyses (PRISMA) statement [18]. It includes the following steps:

1. Identifying the purpose and intended goals of the review (Section 3).

2. Search strategy (Section 3.1).

3. Screening for inclusion (Section 3.2).

4. Screening for exclusion (Section 3.3).

5. Data extraction (Section 4).

6. Analysis (Section 4)

7. Discussion (Section 5).

8. Writing the review.

\subsection{Search Strategy}

The literature search was performed using the Scopus and Web of Science databases. They are multidisciplinary databases and some of the largest abstract and indexing databases of peer-reviewed scientific journals, books, and conference proceedings. Since the covered sources span many individual databases (e.g., citations that are in EMBASE are also in Scopus), it was decided to search only Scopus and Web of Science directly instead of a handful of smaller databases. To perform the search, a set of search terms related to the usage of machine-learning algorithms for PU prevention were identified. Thus, after some initial experiments, and considering a previous analysis of some literature studies, three groups of terms were identified that should be considered in the search. First, terms related to PU and other terms that are used synonymously. Second, terms related to artificial intelligence (AI) algorithms. Third, terms that associate the fact that the patient is lying on a bed. Thus, the complete string for the search was defined as follows:

(bedsore OR bed sore OR decubitus ulcer OR decubitus sore OR pressure ulcer OR pressure sore OR pressure injury)

AND

(artificial intelligence OR computational intelligence OR machine learning OR machine intelligence OR neural network OR support vector machine OR naive bayes OR bayesian learning OR support vector OR random forest OR deep learning)

AND

(bed OR bedridden)

The search was performed in June 2021 and resulted in 59 studies (48 after removing duplicates).

Additionally, one more study was identified through other sources.

\subsection{Screening for Inclusion}

Two reviewers examined the collected set of studies by title and, when necessary, the abstract, to decide whether, for the purposes of the review, they were worth reading further or if they should be excluded. This screening considered that studies should only be included in the review if they met the following criteria: (1) Studies that presented ML-based algorithms to identify patients at risk and provide actions/recommendations for helping in PU prevention; (2) studies that were published in a scientific peer-reviewed publication; (3) studies that were published after 2010. Considering the significant advances in recent years, we assumed that the period between 2010 and 2021 would allow us to 
obtain the most relevant articles and that these articles would allow us to study the most representative approaches. At the same time, we try to avoid analyzing algorithms or approaches that are currently uncommon or that do not fit the current reality regarding pressure-ulcer prevention. We additionally required: (4) studies that were written in English, and (5) studies with full text available. Studies that did not meet all these criteria were excluded.

At this stage, the reviewers did not judge the quality or evaluate the information found in each study. Criterion (1) was determined merely by reviewing the title and, when necessary, the abstract.

After applying criteria 2-5, 16 studies (9 published before 2011 and 7 reviews, notes and others) were excluded. This resulted in 33 studies.

In criterion (1) the focus was on predictive models that were used to identify risk factors or to determine when a patient is at risk or specific measures need to be taken, e.g., repositioning, nutrition, or skin care. After applying this criterion, analyzing the title and abstract, 21 studies were excluded. Of those that were excluded, 12 had the objective of recognizing postures and, in 4 of them, the focus was on image recognition. Five more studies were removed for other reasons. This resulted in 12 studies.

\subsection{Screening for Exclusion}

The final list of studies was assessed by two reviewers to validate whether they should be included in the quantitative and qualitative analysis. At this stage, each reviewer analyzed the full text of each study to assess in detail the purpose, strategy, and outcomes of each study. Studies that did not present enough information about the used algorithm or that did not have an outcome were excluded. Review papers, opinion papers and extended abstracts were also excluded.

After full text analysis, five more studies were excluded. Two of these were focused on recognizing body posture and movements and another on predicting bed inclination. Two other studies were removed either because they are still preliminary studies or because they lack important information.

Thus, seven studies were included in the review after the literature search. Despite the low number, a significant proportion of the articles obtained after the initial search, while presenting ML algorithms for PU prevention or treatment, did not provide any type of recommendation or alert to help prevent PU. This seems to indicate that, although there is a considerable number of studies that address the use of ML for the prevention and treatment of PU, there are still few that, based on the state of each patient, provide some type of recommendation/alert to help healthcare professionals in their tasks.

\subsection{Results}

As presented in Figure 1, after searching for the literature, 49 studies were obtained (after removing 11 duplicates), and after applying the inclusion criteria identified in Section 3.2, 16 studies were excluded, resulting in 33 studies. These studies were evaluated in terms of title and abstract, resulting in the exclusion of 21 studies. The full-text evaluation of the remaining 12 studies was performed, excluding 5 studies that did not match the defined inclusion criteria, did not present sufficient information about the algorithm used or were lacking an outcome. The remaining 7 studies were presented in the qualitative and quantitative synthesis. 


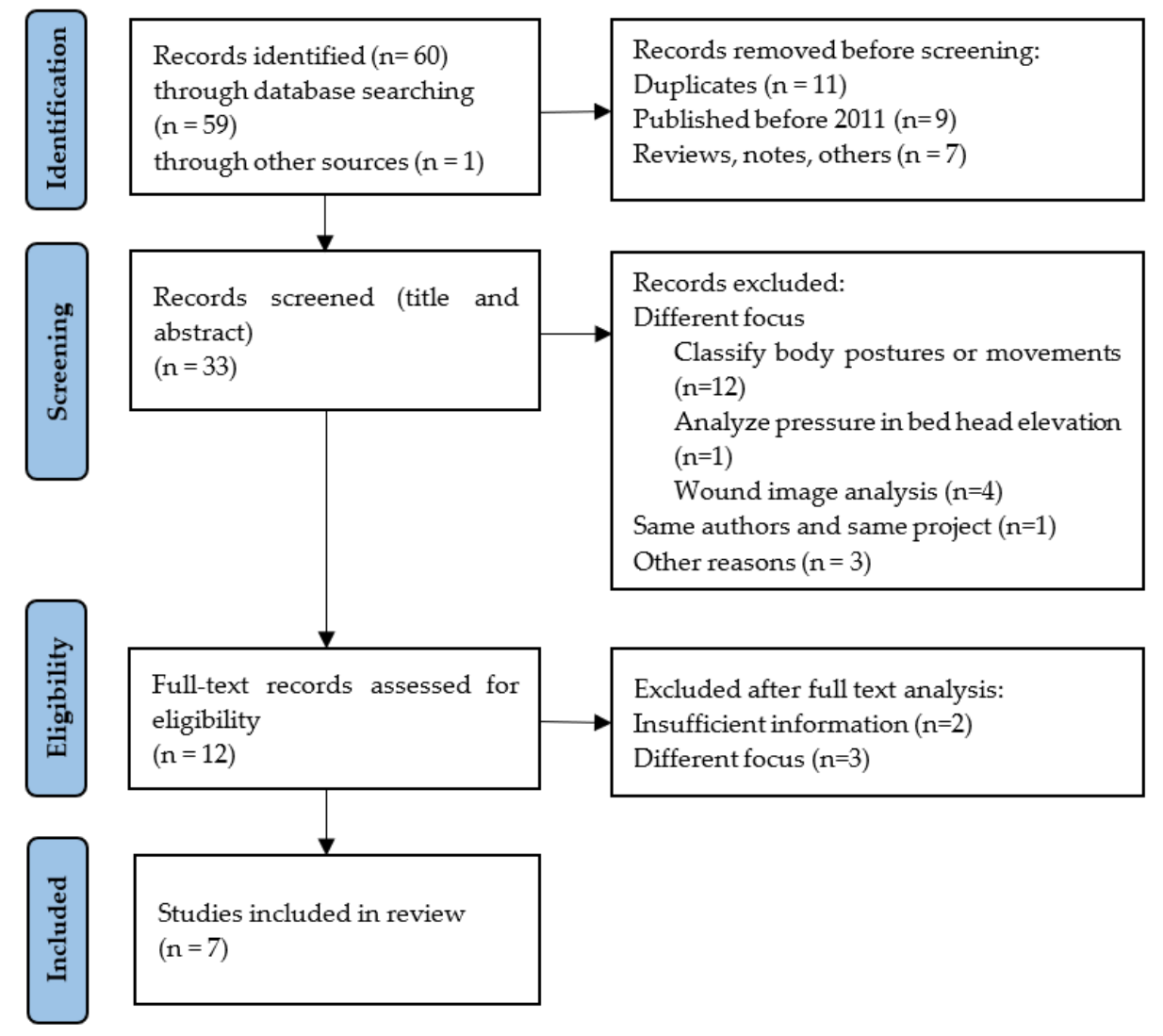

Figure 1. The PRISMA 2020 flow diagram (adapted from [18])—identification of studies.

\subsection{Characteristics of Included Studies}

The seven studies that were included in the review were published between 2011 and 2021 and were undertaken in China (three studies), United States (two studies), Mexico (one study), and Thailand (one study).

All the studies included in the review address the use of ML-based algorithms to identify patients at risk and provide actions/recommendations for help in PU prevention. However, not all of them presented the same level of detail and there are even some studies that approached this issue in a very superficial way. Some advocate for the use of alarms and warnings to alert caregivers that some action will be needed, others only predict the risk of patients developing PU.

\section{Data Extraction and Data Analysis}

Data were extracted from all identified studies using a predefined format. Data extracted included: year of publication, country, type of document, aim, algorithm used, input variables, study outcomes, and findings.

Two reviewers extracted the information. Any disagreements were resolved via discussion. Table 3 identifies the types of data to be extracted.

The characteristics of the included studies are summarized in Table 4.

An identification of the algorithm used in the predictive model and a description of its relevant inputs and outputs is presented in Table 5.

Table 6 presents some details of the ML algorithm that are used in the predictive models. 
Table 3. Type of data to be extracted.

\begin{tabular}{cc}
\hline Item & Description \\
\hline Year of publication & Year of publication \\
Country & Country/territory \\
Document type & Aim \\
AI algorithm & Document type (article, conference paper, book chapter) \\
Input & Main goal of the proposed system \\
Predictive model & AI-based algorithm used in the proposed system \\
Results and findings & Input variables \\
& Details of the predictive model \\
\hline
\end{tabular}

Table 4. Identification and goal of the study $(n=7)$.

\begin{tabular}{|c|c|c|c|c|}
\hline Study & Year & Country & Type & Summary/Aim \\
\hline [6] & 2011 & USA & Conference paper & $\begin{array}{l}\text { Collect information from several sensors incorporated into the bed, } \\
\text { analyze the data to create a time-stamped, whole-body pressure } \\
\text { distribution map, and command the bed's actuators to periodically } \\
\text { adjust its surface profile to redistribute pressure over the entire body. } \\
\text { Predictions are used for issuing an early warning (alert) flag } \\
\text { indicating the existence of high risk of developing an ulcer and control } \\
\text { command/data for pressure redistribution around high-risk limbs. }\end{array}$ \\
\hline [7] & 2015 & Mexico & $\begin{array}{l}\text { Journal } \\
\text { article }\end{array}$ & $\begin{array}{c}\text { Program a sequence of movements of a robotic bed that are executed } \\
\text { automatically through electric actuators in response to the pressure } \\
\text { distribution of a patient on the bed. }\end{array}$ \\
\hline [8] & 2018 & China & $\begin{array}{l}\text { Journal } \\
\text { article }\end{array}$ & $\begin{array}{l}\text { Develop an artificial neural network (ANN) model for predicting } \\
\text { surgery-related pressure injury (SRPI) in cardiovascular surgical } \\
\text { patients. Four risk factors were included in the ANN model: length of } \\
\text { surgery, disease category, age, and corticosteroids. The ANN model } \\
\text { had a moderate prediction value for SRPI. }\end{array}$ \\
\hline [9] & 2020 & China & $\begin{array}{l}\text { Journal } \\
\text { article }\end{array}$ & $\begin{array}{l}\text { Predict SRPI in cardiovascular surgical patients. } \\
\text { An ML model using the XGBoost algorithm is used for SRPI } \\
\text { prediction in patients undergoing cardiovascular surgery. As } \\
\text { potential risk factors, they use demographic characteristics, SRPI } \\
\text { information, and others. They find that the most important predictors } \\
\text { are duration of surgery, patient weight, duration of the } \\
\text { cardiopulmonary bypass procedure, patient age, and disease category. }\end{array}$ \\
\hline [10] & 2018 & USA & $\begin{array}{l}\text { Journal } \\
\text { article }\end{array}$ & $\begin{array}{c}\text { Develop a model for predicting risk of PU among surgical critical care } \\
\text { patients. A random forest model is used to predict stage } 1 \text {, stage } 2, \\
\text { and greater injuries, by using the testing set to evaluate } \\
\text { classifier performance. }\end{array}$ \\
\hline [11] & 2019 & Thailand & Conference paper & $\begin{array}{l}\text { Design a system to relieve the work for the caretaker of a bedridden } \\
\text { patient, consisting of three parts: sleep data collection where a } \\
\text { computer, connected to Kinect, will continuously monitor the patient; } \\
\text { sleep posture analysis, which will determine the postures of the } \\
\text { patient; and sleep notification part which will notify users of the } \\
\text { current state of the patient. Three ML algorithms were applied to } \\
\text { compare their performance: decision tree (DT), neural network (NN), } \\
\text { and support vector machine (SVM). }\end{array}$ \\
\hline [12] & 2020 & China & $\begin{array}{l}\text { Journal } \\
\text { article }\end{array}$ & $\begin{array}{l}\text { Construct a patient service system based on quality function } \\
\text { deployment (QFD) in the IoT environment. With the support of IoT } \\
\text { technology, a deep-learning algorithm and a QFD method were used } \\
\text { to build a patient service model to achieve detection and nursing } \\
\text { reminders of patients' physical conditions. }\end{array}$ \\
\hline
\end{tabular}


Table 5. Details of the algorithm: inputs and outputs $(n=7)$.

\begin{tabular}{|c|c|c|c|c|c|}
\hline Study & Participants & Algorithm & Inputs & Type of Results & Findings \\
\hline$[6]$ & $\begin{array}{l}\text { NA } \\
\text { simulation }\end{array}$ & SVM & $\begin{array}{l}\text { Pressure map (sensor matrix in the bed), level of } \\
\text { moisture, temperature, mobility / activity, and } \\
\text { blood pressure. }\end{array}$ & $\begin{array}{l}\text { Recognize body posture and predict } \\
\text { the risk associated with various limbs } \\
\text { and the whole body. } \\
\text { Alert caregivers to intervene at an } \\
\text { early stage. }\end{array}$ & $\begin{array}{l}\text { This system augments the ability } \\
\text { of a care giver, allowing them to } \\
\text { provide better care to more } \\
\text { patients in less time (without } \\
\text { clinical evaluation). }\end{array}$ \\
\hline [7] & - & SVM & Pressure map from sensor array. & $\begin{array}{l}\text { Recognize body posture (four } \\
\text { positions) and program a sequence of } \\
\text { movements of a robotic bed. }\end{array}$ & \\
\hline [9] & 149 & XGBoost & $\begin{array}{c}\text { Electronic health records: } \\
\text { demographic characteristics (age, gender, weight, and } \\
\text { disease category), SRPI information (number of ulcers, } \\
\text { ulcer severity, anatomical location, corticosteroid } \\
\text { information included administration, type of drug } \\
\text { used, drug dosage, and frequency of drug } \\
\text { administration), and risk factors (use of vasoactive } \\
\text { drugs, experiencing hypotensive periods, hemoglobin } \\
\text { level, albumin level, and use of corticosteroids). }\end{array}$ & $\begin{array}{l}\text { Predict SRPI in cardiovascular } \\
\text { surgical patients. }\end{array}$ & $\begin{array}{l}\text { The most important variables } \\
\text { based on the mean decrease } \\
\text { accuracy were, in descending } \\
\text { order: duration of surgery, body } \\
\text { weight, duration of } \\
\text { cardiopulmonary bypass } \\
\text { procedure, age, and disease } \\
\text { category. }\end{array}$ \\
\hline [10] & 6376 & $\mathrm{RF}$ & $\begin{array}{c}\text { Electronic health records: } \\
\text { hypotension, level of consciousness (Glasgow Coma } \\
\text { Scale), delirium, oxygenation, severity of illness, } \\
\text { temperature, vasopressor medication received, body } \\
\text { mass index at admission, laboratory value (albumin, } \\
\text { creatinine, glucose, hemoglobin, lactate, prealbumin), } \\
\text { surgical time. }\end{array}$ & $\begin{array}{l}\text { Predict SRPI in surgical critical } \\
\text { care patients. }\end{array}$ & $\begin{array}{l}\text { Variables deemed most } \\
\text { important in the algorithm were, } \\
\text { in descending order: body mass } \\
\text { index, hemoglobin level, } \\
\text { creatinine level, time required for } \\
\text { surgery, and age. }\end{array}$ \\
\hline [11] & & $\begin{array}{l}\text { NN } \\
\text { SVM } \\
\text { DT }\end{array}$ & $\begin{array}{l}\text { Patients' movement captured through Kinect. Input } \\
\text { data represents the body joints of the patient. }\end{array}$ & $\begin{array}{l}\text { Recognize body posture. } \\
\text { Alert caregivers to intervene when the } \\
\text { patient is absent or when he or she } \\
\text { stays too long in the same position. }\end{array}$ & NN performs better. \\
\hline [12] & $\begin{array}{l}\text { NA } \\
\text { simulation }\end{array}$ & Deep learning & $\begin{array}{l}\text { Firstly, the personal information of the elderly or } \\
\text { patients is logged in; secondly, the physiological } \\
\text { parameters of the human body are automatically } \\
\text { collected, and the physiological signals of the elderly } \\
\text { or patients are collected by sensors. }\end{array}$ & $\begin{array}{c}\text { The monitoring terminal send out a } \\
\text { warning of abnormal physiological } \\
\text { indicators. }\end{array}$ & $\begin{array}{l}\text { If a nurse can look after four } \\
\text { patients in the traditional way, } \\
\text { with the Service System, he or } \\
\text { she can look after six or more } \\
\text { patients. Reduce the incidence of } \\
\text { a bedsore ( } 22.6 \% \text { to } 4.1 \%) \text { and } \\
\text { increase the average patrol from } \\
\quad 30 \text { min to } 2 \mathrm{~h} \text {. }\end{array}$ \\
\hline
\end{tabular}


Table 6. Details of the predictive model $(n=7)$.

\begin{tabular}{|c|c|c|c|c|c|}
\hline Study & Algorithm & Goal & Training & Testing & Accuracy \\
\hline [6] & SVM & Posture recognition and predict the risk associated & - & - & $97.7 \%$ \\
\hline [7] & SVM & Posture recognition and bed movements & - & - & $99.7 \%$ \\
\hline [8] & ANN & Predict surgery-related pressure injury & $73.2 \%$ & $26.8 \%$ & $81.5 \%$ \\
\hline [9] & XGBoost & Predict surgery-related pressure injury & - & - & $80.6 \%$ \\
\hline [10] & RF & Predict surgery-related pressure injury & $67 \%$ & $33 \%$ & $79 \%$ \\
\hline \multirow{3}{*}[11]{} & NN & Posture recognition and alerts & - & - & $100 \%$ \\
\hline & SVM & Posture recognition and alerts & - & - & $100 \%$ \\
\hline & DT & Posture recognition and alerts & - & - & $93.3 \%$ \\
\hline [12] & Deep Learning & $\begin{array}{l}\text { Send out a warning of abnormal } \\
\text { physiological indicators }\end{array}$ & - & - & $98.53 \%$ \\
\hline
\end{tabular}

\section{Discussion and Findings}

Database searching identified 49 distinct studies (after excluding duplicates) related to the use of ML technologies in PU prevention for bedridden people. Of these studies, 42 were not included in the review, as they did not meet the inclusion criteria. The main reason for the exclusion of these studies was that they did not focus on the use of ML-based algorithms to identify patients at risk, nor provide actions/recommendations to help in PU prevention and treatment. In most of these cases the studies used ML algorithms and were applied to the study of PU in bedridden people. However, they were focused neither on predicting the risk of developing PU, nor on generating any kind of recommendations and/or alarms to alert health professionals about situations that needed their attention. Additionally, they did not consider the intrinsic and extrinsic predisposing factors to produce recommendations or alerts that are customized according to the current state of the patient. In fact, 14 of the excluded studies focused on the classification of body postures or movements (e.g., [19-26]), which represents a very important subject in the monitoring of PU. Four of the excluded studies (e.g., [27-30]) addressed wound image analysis to characterize or classify PU. Despite describing interesting works, these studies did not propose any type of actions or consequences related to the postures and movements identified, or the stage (i.e., pressure ulcers classification according to the level of tissue damage), to improve the prevention and treatment of PU.

Studies included in the review ranged from 2011 to 2020, one being from 2011, one from 2015, two from 2018, one from 2019, and three from 2020, of which three were from China, two from USA, one from Mexico, and one from Thailand, as can be seen in Table 4. These studies are aimed at recognizing body posture in bed (e.g., [6,7,11,12]) and identifying potential risk of developing pressure ulcers (e.g., [8-10]) and providing some type of alerts or recommendations.

Generally, the studies proposed solutions based in systems with tiers or components: data acquisition; data analysis and production of some support for well-informed clinical decision-making (e.g., a warning alarm for patient position).

Regarding data acquisition, three methods were identified. Data was collected from: demographic and pertinent clinical information (e.g., age, gender, diseases, etc.); the patient's medical history; and sensors in the patient's environment, in most cases attached to the beds or to the mattresses. From the acquired data, algorithms were applied to build predictive models to support healthcare professionals' decisions/actions.

Data analysis was performed using distinct algorithms. According to Tables 5 and 6, neural networks were used in two studies $[8,11]$, support vector machines (SVM) were 
used in three studies [6,7,11], and XGBoost [9], RF [10], DT [11] and deep learning [12] were each used in one study.

Some studies did not describe how they used the algorithm output, although other studies referred alerts or warnings to caregivers to intervene when the patient was absent or when he or she stayed in the same position for too long.

The studies analyzed in Section 3, their approaches, results, and findings, allow us to highlight some factors that were reported to have a significant influence on the accuracy of the ML algorithms that were used. Those most frequently reported include information related to intrinsic and extrinsic predisposing factors, namely: demographic (age and gender); medical history (time for surgery, creatinine level, hemoglobin level, body mass index and comorbidities, which can lead to other complications such as anemia of chronic disease, recurrent infection, polypharmacy, and hospitalization) and information collected by sensors (position, movements, and moisture).

\section{Challenges and Opportunities}

The use of ML technologies creates new opportunities to develop a large range of solutions for risk assessment and management of preventive treatments of PU, which could go far beyond what is done nowadays. These solutions may help in monitoring and treatment, making alerts and recommendations that are relevant to support healthcare professionals' decisions/actions, reducing their burden, contributing to an improvement in the quality of life for the patient, and to a decrease in health service costs. However, to achieve this, there are several challenges that need to be overcome, and that raise substantial opportunities for future research.

Many of the works that use ML technologies to address risk assessment, diagnosis and treatment in PU, propose solutions that are based on systems with three tiers: data acquisition; data analyses; and production of some support for well-informed clinical decision-making. Most of them specifically address one of these tiers. However, recommendations and alerts for decision support for healthcare professionals will only be useful if they also include input information from other tiers, such as data acquisition and data analysis. This issue was also identified in [13] and represents an important opportunity for future research.

Several predictive models have only presented alerts as output, which are determined as a function of the patient's movement or posture. They are, for most of the models, simple rules that do not consider many other intrinsic factors of each patient. Furthermore, many studies do not describe in detail these outputs, alerts, and/or recommendations, and do not present any study of their impact on the activity of healthcare professionals or on the wellbeing of the patients. There is a clear need for more in-depth assessment to evaluate the real impact of using these outputs.

Another important challenge is that prediction models must include information about both intrinsic and extrinsic predisposing factors. Most of the proposed prediction models only use information from extrinsic factors. For example, many of the analyzed studies were excluded because they did not match the defined inclusion criteria, since they only used information from sensors installed on the bed or on the mattresses as inputs for the prediction algorithms. Other studies only used information about the patient's medical record as inputs. All these studies show interesting results. However, to have recommendations or alerts that are relevant to the activity of health professionals, these inputs must be analyzed together. This is the only way to obtain recommendations that are personalized according to the specific situation of each patient. Two patients with different levels of tissue damage from PU should have customized recommendations or alerts, specific to their condition, even if they present similar movements and posture recognition.

\section{Strengths and Limitations of this Review}

This review follows the PRISMA methodology. It provides a comprehensive review of the scientific literature concerning the use of ML algorithms for PU prevention. This review 
is important because it presents a characterization of the approaches that use ML for PU prevention, namely the algorithms used, the parameters used as input data, the results they obtained, and how these results can help health professionals in PU prevention and treatment. Furthermore, this study identifies a set of opportunities that might be explored in future research. However, it has also some limitations.

The search for literature was carried out using two databases (Scopus and Web of Science). Although these databases cover several domains and span many individual databases (e.g., citations that are in EMBASE are also in Scopus), this decision may have influenced the number of relevant articles obtained. The use of other databases such as: IEEE Xplore, ACM Digital Library, BMC, and PubMed, could possibly have increased the number of analyzed articles and contributed to improve the overall analysis. Also, the search strategy was deemed to restrict the number of non-relevant articles (articles that were published many years ago, articles that are too general or articles that do not focus on the research objectives). Additionally, only articles written in English were included in the study. These options may have discarded relevant articles, e.g., those written in languages other than English. Another limitation is related to the lack of pertinent information in some of the articles. For example, less exhaustive wording of the ML algorithms used.

These limitations may have significantly affected the number of records obtained and may have had some impact on the retrieval of relevant articles. Consequently, this systematic review was limited by the low number of records analyzed and the eligibility of heterogeneous studies. Also, in some cases, they may have influenced the data extraction and data analysis. However, these constraints did not have a significant effect on the discussion and conclusions.

\section{Conclusions}

The present study, a systematic review based on PRISMA methodology, took a different approach from all previous empirical work on this topic, in trying to establish relations among three axles: intrinsic and extrinsic patient data; real-time data acquisition for bedridden patients; and ML algorithms applied to these two types of data. This approach was chosen with an aim to improve healthcare quality for PU patients and contribute to minimize burnout in healthcare professionals.

In healthcare services, burnout is understood as specific occupational stress, resulting from the demanding relationships between healthcare professionals and their patients, and the lack of availability of personal resources. The improvement of personal health practice can be supported by systems that use the most complete set of data and ML, so that they can provide information for acting according to individual diagnoses.

New methods, like ML, are needed to achieve accurate predictions. The operational definition for data acquisition for these systems to improve care of PU patients has two dimensions: intrinsic and extrinsic data, more specifically individual patient characteristics (intrinsic) and retrieved from the environment (extrinsic) - based on healthcare professionals' observation; and humanless data acquisition, such as obtained by sensors attached to a mattress. Rules applied to the combination of these two types of data can produce indicators or alerts for caregivers that act like a facilitator for healthcare practice, and consequently avoid burnout.

The results of this review show that no relevant studies were found that combine the two data dimensions mentioned above. Systems that use ML to produce effective alerts for caregivers other than a basic signal or an alert beep were also not found. Moreover, there is a clear need for more in-depth assessment to evaluate the real impact of using these outputs.

We recommend a larger study to develop a system that combines the two data dimensions with ML, feeding caregivers not only with signal alerts but also with a diagnosis proposal (such as body repositioning to avoid the development of ulcers), for each patient according to his or her individual conditions. 
Author Contributions: Conceptualization, methodology, validation, writing-review and editing: F.R., F.F., J.M., A.S., R.D., O.S. Formal analysis, investigation, writing-original draft preparation: F.R., F.F. All authors have read and agreed to the published version of the manuscript.

Funding: This work was carried out under the SensoMatt project, grant agreement no. CENTRO-010247-FEDER-070107, co-financed by European Funds (FEDER) by CENTRO2020.

Institutional Review Board Statement: Not applicable.

Informed Consent Statement: Not applicable.

Data Availability Statement: Not applicable.

Conflicts of Interest: The authors declare no conflict of interest.

\section{References}

1. Bluestein, D.; Javaheri, A. Pressure ulcers: Prevention, evaluation, and management. Am. Fam. Physician 2008, 78, 1186-1194.

2. Garcia, A.D.; Thomas, D.R. Assessment and management of chronic pressure ulcers in the elderly. Med. Clin. N. Am. 2006, 90, 925-944. [CrossRef] [PubMed]

3. Haesler, E. (Ed.) National Pressure Ulcer Advisory Panel, European Pressure Ulcer Advisory Panel and Pan Pacific Pressure Injury Alliance. In Prevention and Treatment of Pressure Ulcers: Quick Reference Guide; Cambridge Media: Osborne Park, Australia, 2014.

4. National Institute for Health and Care Excellence Pressure Ulcers. Quality Standard [QS89]. Available online: https://www.nice. org.uk/guidance/qs89 (accessed on 12 July 2021).

5. Jaul, E. Assessment and Management of Pressure Ulcers in the Elderly. Drugs Aging 2010, 27, 311-325. [CrossRef] [PubMed]

6. Yousefi, R.; Ostadabbas, S.; Faezipour, M.; Nourani, M.; Ng, V.; Tamil, L.; Bowling, A.; Behan, D.; Pompeo, M. A smart bed platform for monitoring \& Ulcer prevention. In Proceedings of the 2011 th International Conference on Biomedical Engineering and Informatics (BMEI), Shanghai, China, 15-17 October 2011; Volume 3, pp. 1362-1366. [CrossRef]

7. Vázquez-Santacruz, E.; Cruz-Santos, W.; Gamboa-Zúñiga, M. Design and Implementation of an Intelligent System for Controlling a Robotic Hospital Bed for Patient Care Assistance. Comput. Y Sist. 2015, 19, 467-474. [CrossRef]

8. Chen, H.-L.; Yu, S.-J.; Xu, Y.; Yu, S.-Q.; Zhang, J.-Q.; Zhao, J.-Y.; Liu, P.; Zhu, B. Artificial Neural Network: A Method for Prediction of Surgery-Related Pressure Injury in Cardiovascular Surgical Patients. J. Wound Ostomy Cont. Nurs. Off. Publ. Wound Ostomy Cont. Nurses Soc. 2018, 45, 26-30. [CrossRef]

9. Cai, J.-Y.; Zha, M.-L.; Song, Y.-P.; Chen, H.-L. Predicting the Development of Surgery-Related Pressure Injury Using a Machine Learning Algorithm Model. J. Nurs. Res. 2020, 29, e135. [CrossRef] [PubMed]

10. Alderden, J.; Pepper, G.A.; Wilson, A.; Whitney, J.D.; Richardson, S.; Butcher, R.; Jo, Y.; Cummins, M.R. Predicting Pressure Injury in Critical Care Patients: A Machine-Learning Model. Am. J. Crit. Care Off. Publ. Am. Assoc. Crit. Nurses 2018, 27, 461-468. [CrossRef]

11. Srisrisawang, N.; Narupiyakul, L. Sleep Posture Recognition for Bedridden Patient. In Proceedings of the Mobile and Wireless Technology 2018; Kim, K.J., Kim, H., Eds.; Springer: Singapore, 2019; pp. 79-87.

12. Huang, A.; Cao, J.; Zhang, H. Construction of patient service system based on QFD in internet of things. J. Supercomput. 2021, 77, 2155-2171. [CrossRef]

13. Jiang, M.; Ma, Y.; Guo, S.; Jin, L.; Lv, L.; Han, L.; An, N. Using Machine Learning Technologies in Pressure Injury Management: Systematic Review. JMIR Med. Inform. 2021, 9, e25704. [CrossRef]

14. Shi, C.; Dumville, J.C.; Cullum, N. Evaluating the development and validation of empirically-derived prognostic models for pressure ulcer risk assessment: A systematic review. Int. J. Nurs. Stud. 2019, 89, 88-103. [CrossRef]

15. Tulloch, J.; Zamani, R.; Akrami, M. Machine Learning in the Prevention, Diagnosis and Management of Diabetic Foot Ulcers: A Systematic Review. IEEE Access 2020, 8, 198977-199000. [CrossRef]

16. Marchione, F.G.; Araújo, L.M.Q.; Araújo, L.V. Approaches that use software to support the prevention of pressure ulcer: A systematic review. Int. J. Med. Inform. 2015, 84, 725-736. [CrossRef] [PubMed]

17. Araujo, S.M.; Sousa, P.; Dutra, I. Clinical Decision Support Systems for Pressure Ulcer Management: Systematic Review. JMIR Med. Inform. 2020, 8, e21621. [CrossRef] [PubMed]

18. Page, M.J.; McKenzie, J.E.; Bossuyt, P.M.; Boutron, I.; Hoffmann, T.C.; Mulrow, C.D.; Shamseer, L.; Tetzlaff, J.M.; Akl, E.A.; Brennan, S.E.; et al. The PRISMA 2020 statement: An updated guideline for reporting systematic reviews. BMJ 2021, $372, \mathrm{n} 71$. [CrossRef] [PubMed]

19. Barsocchi, P. Position Recognition to Support Bedsores Prevention. IEEE J. Biomed. Health Inform. 2013, 17, 53-59. [CrossRef] [PubMed]

20. Matar, G.; Lina, J.-M.; Kaddoum, G. Artificial Neural Network for in-Bed Posture Classification Using Bed-Sheet Pressure Sensors. IEEE J. Biomed. Health Inform. 2020, 24, 101-110. [CrossRef]

21. Baran Pouyan, M.; Ostadabbas, S.; Nourani, M.; Pompeo, M. Classifying bed inclination using pressure images. In Proceedings of the 2014 36th Annual International Conference of the IEEE Engineering in Medicine and Biology Society, Chicago, IL, USA, 6-30 August 2014; pp. 4663-4666. [CrossRef] 
22. Rodríguez, A.P.; Gil, D.; Nugent, C.; Quero, J.M. In-Bed Posture Classification from Pressure Mat Sensors for the Prevention of Pressure Ulcers Using Convolutional Neural Networks. In Proceedings of the Bioinformatics and Biomedical Engineering; Rojas, I., Valenzuela, O., Rojas, F., Herrera, L.J., Ortuño, F., Eds.; Springer International Publishing: Cham, Switzerland, 2020; pp. 338-349.

23. Luo, S.; Zhao, C.; Fu, Y. An Intelligent Human Activity Recognition Method with Incremental Learning Capability for Bedridden Patients. In Proceedings of the 2018 15th International Conference on Control, Automation, Robotics and Vision (ICARCV), Singapore, 18-21 November 2018; pp. 1284-1289. [CrossRef]

24. Kosuge, M.; Ishihara, Y.; Takahashi, M. Body pressure prediction for pressure ulcer prevention in a bed head elevation operation. Adv. Robot. 2021, 35, 181-193. [CrossRef]

25. Lee, H.J.; Hwang, S.H.; Lee, S.M.; Lim, Y.G.; Park, K.S. Estimation of Body Postures on Bed Using Unconstrained ECG Measurements. IEEE J. Biomed. Health Inform. 2013, 17, 985-993. [CrossRef]

26. Hung, Y.-W.; Chiu, Y.; Jou, Y.-C.; Chen, W.-H.; Cheng, K.-S. Bed posture classification based on artificial neural network using fuzzy c-means and latent semantic analysis. J. Chin. Inst. Eng. 2015, 38, 415-425. [CrossRef]

27. Veredas, F.J.; Mesa, H.; Morente, L. Efficient detection of wound-bed and peripheral skin with statistical colour models. Med. Biol. Eng. Comput. 2015, 53, 345-359. [CrossRef]

28. Zoppo, G.; Marrone, F.; Pittarello, M.; Farina, M.; Uberti, A.; Demarchi, D.; Secco, J.; Corinto, F.; Ricci, E. AI technology for remote clinical assessment and monitoring. J. Wound Care 2020, 29, 692-706. [CrossRef] [PubMed]

29. Orciuoli, F.; Orciuoli, F.J.; Peduto, A. A Mobile Clinical DSS based on Augmented Reality and Deep Learning for the home cares of patients afflicted by bedsores. Procedia Comput. Sci. 2020, 175, 181-188. [CrossRef]

30. Kavitha, I.; Suganthi, S.S.; Ramakrishnan, S. Analysis of Chronic Wound Images Using Factorization Based Segmentation and Machine Learning Methods. In Proceedings of the 2017 International Conference on Computational Biology and Bioinformatics; Association for Computing Machinery: New York, NY, USA, 2017; pp. 74-78. [CrossRef] 